\title{
Correction to: Sleep medicine in otolaryngology units: an international survey
}

\author{
Giovanni Cammaroto ${ }^{1,2}(0) \cdot$ Giulia Bianchi ${ }^{3} \cdot$ Henry Zhang ${ }^{4} \cdot$ Vik Veer $^{4} \cdot$ Bhik Kotecha $^{4} \cdot$ Ofer Jacobowitz ${ }^{5}$. \\ Marina Carrasco Llatas ${ }^{6} \cdot$ Paula Martínez Ruiz de Apodaca ${ }^{6} \cdot$ Rodolfo Lugo $^{7} \cdot$ Giuseppe Meccariello $^{1}$. \\ Giannicola lannella ${ }^{1} \cdot$ Riccardo Gobbi $^{1}$. Song Tar Toh ${ }^{8} \cdot$ Ying-Shuo Hsu $^{9} \cdot$ Ahmed Yassin Baghat $^{10}$. \\ Jerome R. Lechien ${ }^{2,11}$. Christian Calvo-Henriquez ${ }^{2,12}$. Carlos Chiesa-Estomba ${ }^{2,13}$ - Maria Rosaria Barillari2,14. \\ Badr Ibrahim ${ }^{2,15} \cdot$ Tareck Ayad $^{2,15} \cdot$ Nicolas Fakhry ${ }^{2,16} \cdot$ Paul Hoff $^{17} \cdot$ Eric Rodrigues Thuler $^{18} \cdot$ Lyndon Chan $^{19}$. \\ Chloe Kastoer ${ }^{20}$. Madeline Ravesloot ${ }^{21}$. Heloisa dos Santos Sobreira Nunes ${ }^{22} \cdot$ Andrea De Vito $^{23}$. \\ Filippo Montevecchi ${ }^{1} \cdot$ Claudio Vicini $^{1,2}$
}

Published online: 14 September 2021

(c) Springer Nature Switzerland AG 2021

\section{Correction to: Sleep and Breathing https://doi.org/10.1007/s11325-020-02243-6}

In the original version of this article, author Heloisa dos Santos Sobreira Nunes was unfortunately missed out. The name should be presented as the 28th author of this paper. This is now correctly presented above.

The original article can be found online at https://doi.org/10.1007/ s11325-020-02243-6.

Giovanni Cammaroto

giovanni.cammaroto@hotmail.com

1 Unit of Otolaryngology, Hospital Morgagni Pierantoni, Via Carlo Forlanini 2, 47100 Forlì, Italy

2 Young-Otolaryngologists of the International Federations of Otorhino-laryngological Societies (YO-IFOS), Paris, France

3 Unit of Otolaryngology, University of Ferrara, Ferrara, Italy

4 Unit of Otolaryngology, Head and Neck Surgery, Royal National Throat, Nose and Ear Hospital, London, UK

5 ENT and Allergy Associates, New York, NY, USA

6 Department of Otorhinolaryngology, Dr. Peset University Hospital, Valencia, Spain

7 Department of Otorhinolaryngology, Grupo Medico San Pedro, Monterrey, Mexico

8 Department of Otolaryngology, Singapore General Hospital, Singhealth Duke-NUS Sleep Centre, National University of Singapore, Yong Loo Lin School of Medicine \& Duke-NUS Medical School, Singapore, Singapore

9 Department of Otolaryngology, Shin Kong Wu Ho-Su Memorial Hospital, Taipei, Taiwan

10 Department of Otorhinolaryngology, Alexandria University, Alexandria, Egypt

11 Department of Human Anatomy and Experimental Oncology, Faculty of Medicine, UMONS Research Institute for Health Sciences and Technology, University of Mons (UMons), Mons, Belgium
Publisher's Note Springer Nature remains neutral with regard to jurisdictional claims in published maps and institutional affiliations.

12 Service of Otolaryngology, Travesía de Choupana, Hospital Complex of Santiago de Compostela, Santiago de Compostela, Spain

13 Service of Otolaryngology, Donostia University Hospital, San Sebastian, Spain

14 Department of Mental and Physical Health and Preventive Medicine, University of L. Vanvitelli, Naples, Italy

15 Division of Otolaryngology-Head and Neck Surgery, Centre Hospitalier de l'Université de Montréal, Montreal, QC, Canada

16 Department of Otorhinolaryngology-Head and Neck Surgery, La Conception University Hospital, APHM, Aix Marseille University, Marseille, France

17 Department of Otolaryngology, Head and Neck Surgery, University of Michigan, Ann Arbor, MI, USA

18 Samaritano Hospital, São Paulo, Brazil

19 Illawarra ENTHead andNeck Clinic, Wollongong, NSW, Australia

20 Department of Otorhinolaryngology, Head and Neck Surgery, Antwerp University Hospital, Edegem, Antwerp, Belgium

21 Department of Otorhinolaryngology - Head and Neck Surgery, OLVG, Amsterdam, the Netherlands

22 Nucleus of Otorhinolaryngology and Head and Neck Surgery of São Paulo, São Paulo, Brazil

23 Head \& Neck Department, Ear Nose Throat (ENT) Unit, Santa Maria delle Croci Hospital, Romagna Health Company, Ravenna, Italy 